\title{
O impacto do ensino superior na construção do pensamento de ordem superior
}

\section{The impact of higher education in the development of higher-order thinking}

\author{
Rui Pedro Lopes*, Cristina Mesquita** \\ *Instituto Politécnico de Bragança, IEETA, Portugal, **Instituto Politécnico de Bragança, Portugal; CIEC
}

\begin{abstract}
Resumo
O desenvolvimento económico e social assenta, em grande medida, no conhecimento humano. A forma como as pessoas trabalham e se envolvem requer uma postura de aprendizagem ao longo da vida e de participação. O papel do ensino superior é fundamental, não apenas para estímulo ao desenvolvimento de competências cognitivas, mas também para o desenvolvimento de competências funcionais e sociais. Neste trabalho descreve-se a aplicação de Inquiry Based Learning (IBL) numa turma de Desenvolvimento de Software I num Curso Técnico Superior Profissional para a aprendizagem da programação, verificando-se como os alunos participam e colaboram na construção do seu conhecimento.

Palavras-chave: pensamento de ordem superior; ensino da programação; ensino superior.
\end{abstract}

\begin{abstract}
The economic and social development depends on human knowledge. The way people work and collaborate demand a posture of participation and of life-long learning. Higher education has a fundamental role in the development of these profssionals, not only by stimulating the development of cognitive skills, but also by the development of social and functional skills. The work presented in this paper describes the application of InquiryBased Learning (IBL) in the class of Software Development I of the Superior Technical and Professional program for programming learning, to assess how students participate and collaborate in the development of their own knownledge.
\end{abstract}

Keywords: higher-order thinking; professional skills; higher education.

\section{Introdução}

O conhecimento humano é, atualmente, considerado um fator fundamental para o desenvolvimento económico e social das sociedades modernas. Neste contexto, as Instituições de Ensino Superior (IES) têm um papel fundamental para o sucesso e sustentabilidade da economia do conhecimento. No entanto, nos últimos 40 anos, o ensino superior tem encarado um conjunto de desafios que têm contribuído para a modificação da sua estrutura funcional e organizacional. De facto, as alterações que se têm vindo a verificar têm repercussões no ensino superior. A globalização, a adoção de tecnologia, a expansão do número e diversidade de alunos, as exigências de adaptação dos modelos e abordagens pedagógicas para o desenvolvimento de competências pessoais e profissionais adequadas à configuração socioeconómica provocam alterações na forma como se investiga, ensina e aprende, a nível superior. É esperado que os alunos do ensino superior, além das competências cognitivas, também desenvolvam competências funcionais e sociais, bem como a capacidade de aprender ao longo da vida. Estas são componentes essenciais para o profissional da sociedade pós-industrial.

Neste contexto, pensamento de ordem superior inclui a capacidade de raciocinar criticamente, ser autónomo e proactivo, ter apetência para trabalhar em equipa e capacidade para aprender ao longo da vida.

\section{Pensamento de ordem superior}

Estudos de Bransford, Brown e Cocking (2000) e de Schwartz, Bransford, and Sears (2005) referem que o principal objetivo de aprendizagem passa pelo desenvolvimento de capacidade de adaptação, ou seja, a aplicação de conhecimento e competências de forma flexível e criativa em novas situações. Este conceito vai para lá do aprender a aplicar e da experiência quotidiana em determinados assuntos. Pelo contrário, reúne a capacidade de adaptar, continuamente, as competências essenciais. É, portanto, essencial para os requisitos de uma sociedade em constante aprendizagem e evolução. Para tal, é necessário o desenvolvimento do Pensamento de Ordem Superior (POS).

Há vários conceitos associados ao processo de POS. Em primeiro lugar, os níveis de pensamento não podem ser separados dos níveis de aprendizagem. São interdependentes, requerem múltiplos componentes e apresentam níveis de complexidade distintos. Adicionalmente, os alunos aprendem tanto em contexto educativo (escola) como em contexto sociocultural (comunidade). Os conceitos e vocabulário que aprendem no ano anterior contribuem para que desenvolvimento POS e novo conteúdo no ano seguinte. Em terceiro lugar, o POS envolve uma variedade de processos de pensamento aplicados a situações complexas e com múltiplas variáveis (King, Goodson, \& Rohani, 2009).

De acordo com Dumont, Istance e Benavides (2010), o POS é das características mais valorizadas tanto em contexto profissional como nas relações sociais. Este inclui a capacidade para gerar e processar informação complexa, pensar de forma crítica e sistematicamente, tomar decisões pesando diversas opções, criar questões 
significativas sobre diferentes assuntos, ser flexível e com capacidade de adaptação a nova informação e situações, ser criativo, conseguir justificar e resolver problemas reais, adquirir conhecimento detalhado sobre conceitos complexos, possuir literacia para os media, trabalhar em equipa e comunicar e argumentar de forma persuasiva.

Este conjunto de competências são desenvolvidas por intermédio de estratégias específicas, que se demarcam do modelo tradicional de ensino-aprendizagem e que apelam ao envolvimento profundo do aluno no processo de construção do seu próprio conhecimento.

Neste trabalho é descrita a aplicação de uma metodologia de aprendizagem ativa numa turma de Desenvolvimento de Software I do Curso Técnico Superior Profissional em Desenvolvimento de Software e Administração de Sistemas e é feita uma análise do resultado da sua aplicação para o desenvolvimento de POS.

\section{Inquiry-Based Learning (IBL)}

A Inquiry Based Learning (IBL) tem vindo a destacarse enquanto metodologia de ensino-aprendizagem, constituindo-se como uma estratégia educacional através da qual os alunos/professores seguem processos similares aos utilizados pelos investigadores, no sentido de construir conhecimento relevante (Keselman, 2003). Esta concetualização funda-se na ideia de que a aprendizagem através da descoberta e a construção de significados (Bruner, 2015) cria, em cada aluno, uma compreensão mais profunda e mais permanente (Prestie \& Smith, 2010).

A IBL é considerada como uma abordagem de resolução de problemas, o que implica a utilização de vários procedimentos inerentes a esta estratégia. Conforme se salienta nas perspetivas de Dewey (2007) e Bruner (2008, 2015), esta metodologia enfatiza a participação e responsabilidade do aluno para descobrir o conhecimento do que é novo. Acentua-se o envolvimento e ação dos alunos, mas também a necessidade de organizar de forma clara e sequencial todo o processo investigacional.

$O$ processo investigacional na IBL encontra-se dividido em fases logicamente relacionadas por unidades de ação, que guiam os alunos e focalizam a sua atenção nas características do pensamento reflexivo e científico. Contudo, na revisão da literatura efetuada por Pedaste et al. (2015), que analisaram 32 artigos focalizados na IBL, revelam a existência de diferentes variações dos ciclos de investigação. Numa tentativa de aproximação dos diferentes desenhos metodológicos, os autores identificaram cinco fases distintas:

1. Orientação: estimular a curiosidade acerca de um tópico e lançar um desafio de aprendizagem a partir da formulação de um problema.

2. Concetualização: formular questões baseadas na teoria ou formulação de hipóteses.

3. Investigação: processo de planificar, explorar ou experimentar, recolhendo e analisando os dados, baseados nos procedimentos de investigação.

4. Conclusão: retirar conclusões dos dados recolhidos. Comparação das inferências, a partir da análise dos dados que permitem responder às questões problema.

5. Discussão: apresentar as evidências que se destacam, comunicando-os aos outros e refletindo, em diferentes momentos do ciclo de investigação e aprendizagem.

Estas unidades de ação guiam os alunos e focalizam a sua atenção nas características do pensamento reflexivo e científico.

\section{Metodologia}

O conceito de IBL requer uma metodologia rigorosa de aplicação para se conseguir transpor estas cinco fases para a prática. Adicionalmente, e em paralelo, foi definida uma metodologia de avaliação para permitir aferir o impacto do IBL na construção do POS.

\section{Avaliação}

A avaliação da estratégia pedagógica é efetuada com dois objetivos principais. Por um lado, pretende-se avaliar o impacto que esta tem no processo de desenvolvimento de POS e, por outro lado, incluir os alunos no processo e no desenho das experiências de aprendizagem.

Os alunos devem perceber, desde o início, o que representa a nova abordagem e qual o papel de cada um. Para o efeito, devem refletir sobre a estratégia e serem estimulados e a colocar questões. No início do semestre é feito um questionário, respondido em aula, para averiguar as perceções dos alunos sobre os processos de ensino-aprendizagem. Durante o processo, são aferidos os níveis de satisfação e motivação nos vários momentos da aplicação da IBL. No final, é feita uma avaliação final, comparativa com as perceções iniciais.

A avaliação é feita segundo um processo de investigação-ação, considerando que todos os atores contribuem para o desenho e implementação e para a construção de conhecimento sobre a prática.

Em paralelo com o procedimento de avaliação, a IBL é aplicada como estratégia de ensino-aprendizagem.

\section{Desenho e implementação da IBL}

A IBL é inicia as suas cinco fases com a orientação. Esta ocorre na primeira aula do semestre, começando com a desconstrução da designação da unidade curricular. Os alunos são estimulados a apresentar 10 sinónimos de cada termo do nome da unidade curricular (Desenvolvimento, Software). Segue-se a constituição de equipas. Em conjunto, os alunos selecionam os dois termos mais consensuais e formulam duas frases, que dão lugar à definição e estruturação de um problema. Os temas de investigação são consistentes entre os diversos grupos, incluindo o estudo, discussão e experimentação do ambiente integrado de desenvolvimento, estruturação de módulos do programa, desenho da interface gráfica, identificação de ciclos e elementos de controlo de fluxo, programação paralela (threads) e delegação (associação de classes). Os alunos procuram, com a ajuda do professor, compreender e estudar os problemas. $\mathrm{O}$ desenvolvimento de jogos é um tipo de aplicações que os motiva, pelo que têm vindo a surgir vários temas relacionados com este tipo de projetos. Deste passo, os 
alunos elaboram um relatório, ou guião, que seguem durante a fase seguinte.

A fase de investigação, mais morosa, inclui o estudo dos vários elementos e padrões de desenvolvimento e estruturação de programas e a geração de código. A quarta fase, conclusão, leva os alunos a compararem o resultado final com as suas ideias iniciais, registadas no documento escrito na segunda fase.

Por último, os alunos apresentam e discutem o resultado da sua investigação/programa com o resto da turma, sendo avaliados pelo seu desempenho pelos colegas.

\section{Classificação dos alunos}

No final do semestre, os alunos são classificados numa escala de 0 a 20 , sendo necessário uma nota de 10 para aprovar à unidade curricular.

O modelo de classificação dos alunos consiste em três componentes. A primeira, correspondendo a $20 \%$ da nota, é feita pelo professor e incide sobre o guião desenvolvido pelos alunos na segunda fase. Outra componente, também com $20 \%$, resulta da avaliação pelos pares, em que todos os alunos atribuem uma classificação (escala de 5 valores) a todos os seus colegas. Os últimos $60 \%$ correspondem ao resultado final, que inclui o relatório final e a aplicação.

Em paralelo com o processo de classificação dos alunos e com o decorrer da implementação da IBL, é feita uma avaliação da aplicação da metodologia.

\section{Avaliação}

A aplicação do impacto da IBL foi feita em três momentos. Inicialmente, o levantamento das perceções dos alunos por intermédio de um questionário, o acompanhamento e registo das reflexões do docente ao longo do semestre e um questionário final relativo à satisfação com a metodologia.

\section{Caracterização dos alunos}

A turma, no ano letivo de 2016/2017, funcionou com 22 alunos, dos quais 2 eram mulheres. A média de idades era de 20,4 anos, tendo o mais novo 18 anos e o mais velho 30 anos. No final aprovaram 15 alunos.

\section{Perceções iniciais dos alunos}

A perceção inicial dos alunos foi obtida por intermédio de um questionário, estruturado em 5 dimensões. Estas, além de fazerem uma caracterização do aluno, permitiram averiguar o que os alunos dizem sobre a forma como estudam, como avaliam as suas próprias competências de aprendizagem, ou seja, que atributos académicos consideram mais importantes relativamente aos colegas e a importância que os diversos tópicos, ferramentas e abordagens pedagógicas têm para a aprendizagem da programação.

Relativamente à sua forma de estudar, os alunos reconhecem não ter hábitos de estudo regular (Figura 1). As características menos valorizadas são aquelas que indiciam um trabalho continuado de estudo, ou seja, a ida à biblioteca, estudar antes ou depois da aula, estudar diariamente, entre outras. Por outro lado, as mais valorizadas são as relacionadas com a presença em aula, ou seja, tentar prestar atenção à aula, resolver as tarefas e exercícios práticos e atenção e acompanhamento individual. Revelam, portanto, pouca autonomia e pouca motivação para o estudo.

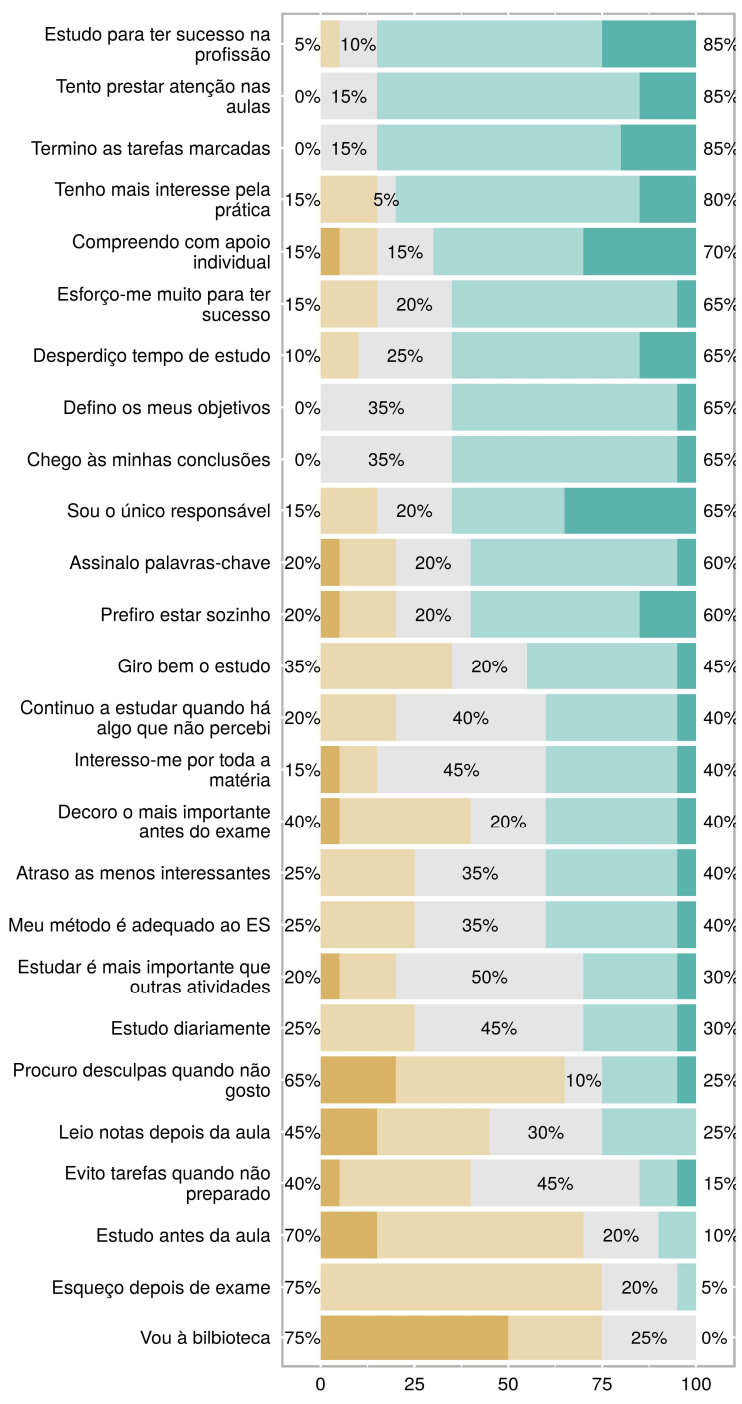

Figura 1. Avaliação da forma de estudar.

Este facto é confirmado quando questionados sobre a importância que atribuem às metodologias pedagógicas (Figura 2). As aulas laboratoriais, os trabalhos e projetos em grupo e os exercícios são mais valorizados que as aulas convencionais ou a leitura orientada.

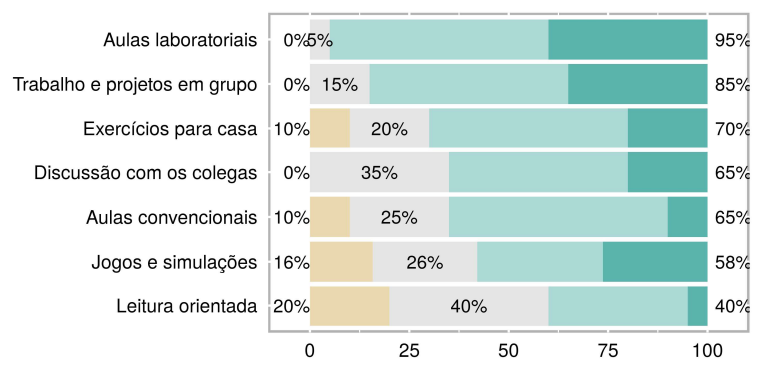

Figura 2. Importância das metodologias pedagógicas. 
Os alunos consideram que é mais importante conseguirem trabalhar em equipa e recorrer a ajuda e aos recursos disponíveis do que organizar o tempo de estudo, saber realizar operações matemáticas ou conseguir escrever textos (Figura 3).

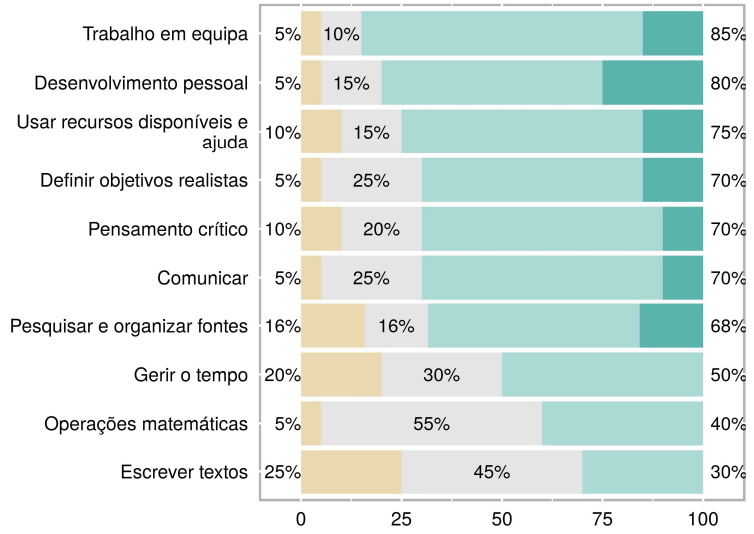

Figura 3. Competências que um aluno deve possuir.

Sobre o tema fundamental da unidade curricular, ou seja, a programação, os alunos têm uma ideia clara dos requisitos e da importância relativa dos assuntos que devem abordar (Figura 4).

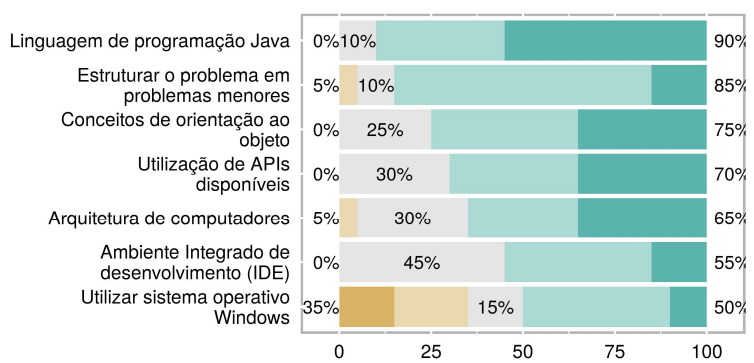

Figura 4. Importância dos assuntos e ferramentas.

Valorizam a sintaxe e semântica da linguagem, a capacidade de estruturar o problema em problemas menores e os conceitos de linguagens orientadas ao objeto, como seria de esperar.

\section{Satisfação com a IBL}

No final do semestre, foi feito um questionário para averiguar o grau de satisfação com a IBL e com os recursos didáticos, bem como com o impacto que esta teve no desenvolvimento de características associadas ao POS. Assim, os alunos consideram que, na generalidade, os recursos foram suficientes e de boa qualidade. No entanto, valorizam menos a quantidade de textos e outros materiais (Figura 5).

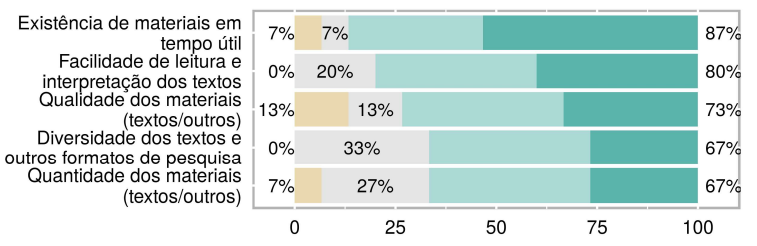

Figura 5. Satisfação com os recursos didáticos.

Os alunos encontram-se satisfeitos com o desempenho do professor, valorizando menos a disponibilidade para os acompanhar durante o desenvolvimento do trabalho (Figura 6).

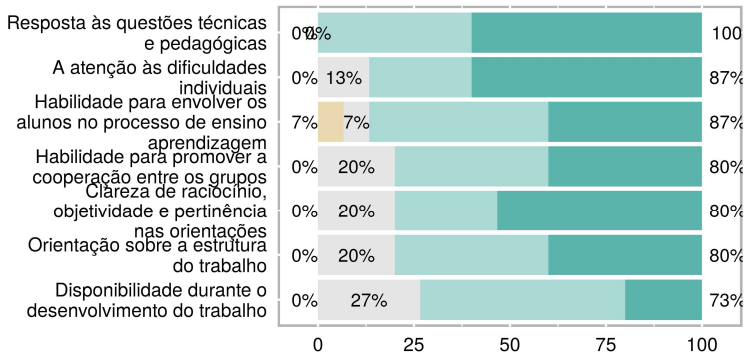

Figura 6. Satisfação com o desempenho do professor.

Os alunos consideram que a metodologia seguida lhes permitiu desenvolver trabalhos motivantes e que lhes causou um maior envolvimento pessoal (Figura 7). Por outro lado, valorizam menos o contributo para o desenvolvimento do espírito crítico, a autonomia e o desenvolvimento de trabalhos sobre assuntos atuais.

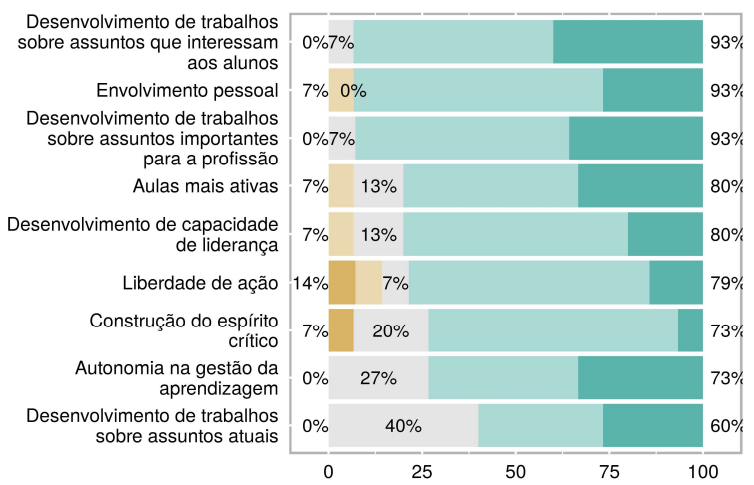

Figura 7. Contributo da IBL para o desenvolvimento do POS.

Relativamente à perceção dos alunos sobre o contributo da estratégia de aprendizagem, apesar de reconhecerem um maior envolvimento pessoal, derivado de trabalharem sobre assuntos que lhes interessa e relevantes para a profissão, valorizam menos as características mais associadas ao desenvolvimento da POS, como gestão da aprendizagem, construção de espírito crítico ou liberdade de ação.

Quando questionados sobre o desenvolvimento de competências transversais, os alunos dizem desenvolver mais a autonomia, a atitude crítica e a interação com os pares, dado trabalharem em conjunto em tarefas mais exigentes ou complexas (Figura 8). 


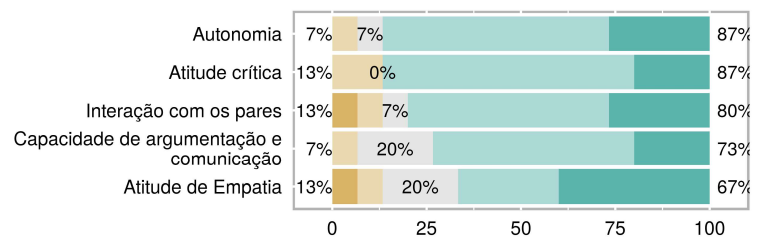

Figura 8. Desenvolvimento de saberes para lá dos académicos.

Nota-se, no conjunto dos dados, que os alunos não demonstram ter ganhado autonomia e motivação para trabalhar e estudar de forma autónoma, necessitando de acompanhamento pelo professor e/ou pelos colegas. $\mathrm{O}$ desenvolvimento do POS só será efetivo se o aluno tiver disponibilidade mental e conseguir aplicar esforço e energia no estudo, reflexão e argumentação com uma forte componente de iniciativa própria. É necessário estimular estas características, o que dificilmente se consegue em apenas uma unidade curricular do curso.

\section{Considerações finais}

A adoção e implementação de metodologias ativas no ensino superior é considerado, pela literatura, uma mais valia para o desenvolvimento de saberes transversais, necessários para um maior comprometimento e participação na vida profissional. A IBL, estruturada em cinco fases que os alunos seguem na construção do conhecimento, foi aplicada numa turma de Desenvolvimento de Software 1 de um Curso Técnico Superior Profissional.

Os alunos apresentam uma perceção inicial adequada e enquadrada com o tema da unidade curricular, reconhecendo as competências chave para o desenvolvimento do pensamento de ordem superior. No entanto, após a aplicação da estratégia e no final do semestre, os alunos apresentam evidências de uma maior motivação e entrega no desenvolvimento de trabalhos e estudo de assuntos que lhes interessa sem reconhecer o contributo que lhes possa ter trazido para o desenvolvimento do POS.

Muito há a fazer do ponto de vista do desenvolvimento do pensamento crítico e raciocínio lógico, sendo necessário uma maior contribuição em outras unidades curriculares. Apesar de se verificarem algumas melhorias, uma unidade curricular não é suficiente para efetivar alterações significativas na forma de pensar e de agir dos alunos.

\section{Referencias}

Bransford, J. D., Brown, A. L., Cocking, R. R., \& others. (2000). How people learn. Washington, DC: National Academy Press. Obtido de http://csun.edu/ SB4310/How\%20People\%20Learn.p df

Bruner, J. S. (2008). Actos de Significado. Edições 70.

Bruner, J. S. (2015). O Processo da Educação. Edições 70.

Dewey, J. (2007). How We Think. Digireads.com. Obtido de http://www.amazon.com/How-We-Think-JohnDewey/dp/1420929976
Dumont, H., Istance, D., Benavides, F., \& OECD (Eds.). (2010). The nature of learning: using research to inspire practice. Paris: OECD.

Keselman, A. (2003). Supporting inquiry learning by promoting normative understanding of multivariable causality. Journal of Research in Science Teaching, 40(9), 898-921. https://doi.org/10.1002/tea.10115

King, F., Goodson, L., \& Rohani, F. (2009). Higher Order Thinking Skills. Center for Advancement of Learning and Assessment. Obtido de http://www.cala.fsu.edu/files/higher_order_thinking_s kills.pdf

Pedaste, M., Mäeots, M., Siiman, L. A., de Jong, T., van Riesen, S. A. N., Kamp, E. T., ... Tsourlidaki, E. (2015). Phases of inquiry-based learning: Definitions and the inquiry cycle. Educational Research Review, 14 , 47-61. https://doi.org/10.1016/j.edurev.2015.02.003

Prestie, A., \& Smith, C. (2010). Technology-Supported Inquiry-Based Learning: Enhancing Teaching and Learning in Secondary Mathematics. Canada: Dr. Stirling McDowell Foundation for Research Into Teaching. Obtido de http://www.mcdowellfoundation.ca/main_medowell/p rojects/research_rep/191technology_supported_inquiry.pdf

Schwartz, D. L., Bransford, J. D., Sears, D., \& others. (2005). Efficiency and innovation in transfer. Transfer of learning from a modern multidisciplinary perspective, 1-51. 